\title{
EXISTENCIA DE INYECTORES EN GRUPOS FINITOS RESPECTO DE CIERTAS CLASES DE FITTING
}

\author{
M.J. IRANZO Y F. PÉREZ MONASOR
}

Abstract

In this paper we prove that all finite groups have $F$-injectors with respect to a saturated and extensible Fitting formation $F$.

Todos los grupos considerados en este trabajo son finitos. Las componentes de un grupo son sus subgrupos subnormales cuasisimples. El producto de todas las componentes de un grupo $G$ es el radical semisimple de $G$, denotado $E(G)$. Las propiedades de las componentes de un grupo pueden encontrarse en $\{[\mathbf{2}$, X.13]).

Para las definiciones y primeras propiedades sobre clases de Fitting e inyectores ver [1].

Denotamos por $N, N^{*}, S, E_{\pi}$ las clases de los grupos nilpotentes, cuasinilpotentes, resolubles, $\pi$-grupos, siendo $\pi$ un conjunto de números primos.

En 1985 encontramos un test para la obtención de $F$-inyectores en cualquier grupo finito. Dicho test es en esencia el siguiente:

1. Existencia de $F$-inyectores de $E(G)$.

2. Sea $I$ un $F$-inyector de $E(G)$. Si $J$ es un $F$-inyector de $N_{G}(I)$, entonces $J$ es un $F$-inyector de $G$.

3. Si $G$ es $F$-constricto, $\left(N_{G}(I)=G\right)$, entonces $G$ posee $F$ - inyectores.

La eficacia de dicho test fue comprobada para el caso en que $N \subseteq F \subseteq N^{*}$, siendo $F$ una clase de Fitting cerrada para cocientes centrales y extensiones centrales. ([4]). Asimismo cuando $F=\left\{G \mid E\left(G / O_{x^{\prime}}(G)\right) \in H, H\right.$ clase de Fitting, $\pi=\operatorname{car} H\}([5])$.

El motivo de este trabajo es probar que sí $F$ es una formación de Fitting saturada y extensible, todo grupo finito admite $F$-inyectores. En este caso Ia última fase del test no parece titilizable de forma inmediata, puesto que no es conocido que todo grupo $F$-constricto tenga $F$-inyectores. Por ello ha sido necesario modificar algo la técnica de la demostración.

To $\mathrm{B}$. Huppert on his sixtieth birthday. 
Lema 1. Sea $F$ un homomorfo de Fitting. Si $G$ es producto directo de grupos simples no abelianos $G_{i}, i=1, \ldots, n$, entonces $G$ posee $F$-inyectores que son exactamente los productos de los subgrupos $F$-maximales de los factores.

Demostración: Vale la misma que la del lema 1 de [4].

En lo que sigue y siempre que no se manifieste lo contrario, $F$ será una formación de Fitting saturada.

Lema 2. Un grupo $G$ posee $F$-inyectores si y solo si los posee $G / Z(G)$.

Demostración: Simple comprobación.

Corolario 1. Dado un grupo cualquieta $G, E(G)$ posee $F$-inyectores.

Demostración: Basta tener en cuenta que $E(G) / Z(E(G))$ es producto directo de grupos simples no abelianos y aplicar los dos lemas anteriores.

Corolario 2. Sea $G=G_{1} G_{2}$ donde $G_{i}$ es semisimple $i=1,2$ y $\left[G_{1}, G_{2}\right]=1$. Si $J$ es un $F$-inyector de $G$, entonces $J=\left(J \cap G_{1}\right)\left(J \cap G_{2}\right)$.

Demostración: Vale una demostración análoga a la del Corolario 2 de [4].

\section{Notas.}

1. Evidentemente $\operatorname{los} F$-inyectores de una componente de $G$ son sus subgrupos $F$-maximales.

2. En virtud de los resultados anteriores, los $F$-inyectores de $E(G)$ son exactamente los productos de los $F$-inyectores de sus componentes.

Teorema. Sea $F$ una formación de Fitting saturada y extensible, entonces todo grupo posee $F$-inyectores.

Demostración: En la demostración del teorema por inđucción, suponemos que la afirmación vale para todos los grupos de orden menor que el orden de un grupo dado $G$. Puesto que es fácil comprobar que si $G / O_{\pi^{\prime}}(G)$ posee $F$ inyectores entonces $G$ posee $F$-inyectores, siendo $\pi=\operatorname{car} F$, podemos suponer que $O_{\pi},\{G)=1$. Sea $I$ un $F$-inyector de $E(G)$. Si $N_{G}(I)<G$, entonces por hipótesis de inducción $N_{G}(I)$ posee $F$-inyectores, sea $J$ uno de ellos. Veamos que $J$ es $F$-inyector de $G$. Sea $N \unlhd \unlhd G$ y $J \cap N \leq W \leq N$ con $W \in F$. Denotemos con $L_{1}$ el producto de las componentes de $G$ contenidas en $N$ y $L_{2}$ el producto de las componentes fuera de $N$. Entonces $E(G)$ es producto central de $L_{1}$ por $L_{2}$ y por el corolario $2, I=\left(I \cap L_{1}\right)\left\{I \cap L_{2}\right)$. Además $W \cap L_{1} \unlhd W$ luego $W \cap L_{1} \in F$ y como $I \cap L_{1} \leq J \cap L_{1} \leq W \cap L_{1}$ y por el carácter $F$-maximal de $I \cap L_{1}$ en $L_{1}$ se sigue $I \cap L_{1}=W \cap L_{1}$ asi $W$ normaliza a $I \cap L_{1}$ y centraliza a $I \cap L_{2}$, luego $W$ normaliza a $I$. Ahora 
tenemos $J \cap N_{N}(I) \leq J \cap N \leq W \leq N_{N}(I)$. Además $N_{N}(I)$ es subnormal en $N_{G}(I)$ luego $J \cap N_{N}(I)$ es $F$-maximal en $N_{N}(I)$, luego $J \cap N=W$, lo que prueba que $J$ es un $F$-inyector de $G$.

Podemos suponer por tanto que $N_{G}(I)=G$. Entonces $E(G)=I R$ con $[I, R]=1, \quad R$ semisimple, así $I \cap R \leq Z(R)$ y como $O_{\pi^{\prime}}(G)=1, Z(R)$ es $\pi$-grupo luego $F$-grupo. Dado que $I \cap R$ es $F$-inyector de $R$ se deduce que $I \cap R=Z(R)$. Por el lema 2 se sigue ahora que 1 es $F$-inyector de $R / Z(R)$ deduciéndose que éste no posee $\pi$-elementos y en consecuencia es un $\pi^{\prime}$-grupo y como $O_{\pi^{\prime}}(G)=1$ y $R$ es semisimple se sigue $R=Z(R)=1$. Asi $E(G) \in F$, es decir $G$ es $F$-constricto [3]. Entonces los normales minimales de $G$ son $F$ grupos. Sea $N$ uno de ellos. Como $|G / N|<|G|$, por inducción $G / N$ posee $F$ -inyectores. Sea $S / N$ uno de ellos, entonces $S \in F$. Supongamos que $G^{*} \unlhd \unlhd G$ y $G^{*} \cap S \leq W \leq G^{*}$ con $W \in F$, entonces $\left(S \cap G^{*}\right) N / N \leq W N / N \leq G^{*} N / N$ y $W N / N \in F$, luego como $S / N \cap G^{*} N / N$ es $F$-maximal en $G^{*} N / N$ se sigue: $\left(S \cap G^{*}\right) N=W N \leq S$ y por tanto $W=S \cap G^{*}$.

\section{Notas.}

1. Cada grupo no resoluble posee al menos dos clases de conjugación de $S$-inyectores.

2. Observar que no se obtiene un resultado análogo para la clase $E_{\pi}$, es decir un grupo con una única clase de conjugación de $E_{\pi}$-inyectores no es necesariamente un $\pi$-grupo. Basta considerar $\pi=\{p\}$ y aplicar teoría de Sylow.

\section{Bibliografía}

1. K. DOERK, T. HaWkes, Finite Soluble Groups, Preprint.

2. B. HUPPERT, N. BlackBURN, Finite Groups III, "Springer-Verlag, Berlin," 1982.

3. M.J. IRANZO, F. PÉREZ MONASOR, $F$-constraint with respect to a Fitting, class, Arch. Math. 46 (1986), 205-210.

4. M.J. IRANZo, Fitting classes $F$ such that all Finite Groups have $F$ -injectors, Israel J. Math. 56 (1986), 97-101.

5. M.J. IRANZO, On the existence of certain injectors in Finite Groups, Communications in Algebra.

\footnotetext{
Departamento de Algebra

Facultad de Ciencias Matemáticas

Univeraidad de Valencia

C/ Doctor Moliner s/n. Burjasot

(Valencia), SPAIN.
}

Rebut el 19 d'Agost de 1986 